01

\title{
Расчет поля в плоскослоистых средах микроэлектроники
}

\author{
() И.А. Конников \\ e-mail: konnikov_i@mail.ru \\ (Поступило в Редакцию 7 февраля 2017 г.) \\ Предложена ориентированная на решение задач микроэлектроники модификация метода расчета гори- \\ зонтальной и вертикальной компонент монохроматического поля, создаваемого в плоскослоистой среде \\ горизонтально протекающим током и описываемого волновым уравнением. Предлагаемое решение ба- \\ зируется на использовании ранее предложенного и апробированного метода эквивалентной постоянной \\ распространения, который позволяет значительно снизить объем вычислений по сравнению с другими \\ методами, основанными на строго динамическом подходе. Рассмотрен ход решения задачи, иллюстрирующей \\ применение предлагаемого метода для области малых расстояний.
}

DOI: 10.21883/JTF.2017.11.45118.2197

\section{Введение. Известные решения}

Решение многих задач технической физики сводится к решению волнового уравнения с последующим вычислением поля реального источника известной формы и размеров через функцию Грина; при этом в качестве физической модели среды, где рассчитывается поле, нередко может быть принята плоскослоистая среда. Как известно $[1,2]$, строгое аналитическое решение волнового уравнения на частоте $\omega>0$ для электромагнитного поля в плоскослоистой среде описывается интегралом на действительной полуоси от комплекснозначной функции, включающей в качестве одного из сомножителей функцию Бесселя первого рода. В англоязычной литературе интегралы этого типа известны как Sommerfeld-type integrals (см., например, $[3,4]$ ); в настоящей работе они именуются $S$-интегралами.

Методы вычисления интегралов указанного типа $(S$-интегралов) хорошо известны. Значения интегралов вычисляются приближенно, для чего применяются различные численные схемы, либо асимптотические методы (чаще всего это - метод перевала), либо их комбинации. Для решения многих прикладных задач, и особенно для проведения научных исследований, такие методы обычно являются вполне приемлемыми. Однако, опуская детальный разбор известных методов вычисления $S$-интегралов, обобщая полученные к настоящему времени результаты, можно констатировать, что для некоторых технических приложений $S$-интегралы все же мало пригодны для практического использования в качестве математической модели электромагнитного поля в плоскослоистой среде. В частности, для решения проектных задач микроэлектроники (например, для количественной оценки перекрестных помех), когда поле требуется вычислять сотни и даже тысячи раз, при использовании компьютеров широко доступного класса $S$-интегралы совершенно непригодны вследствие непомерно высоких затрат машинного времени. Тем же недостатком обладают хорошо известные методы расчета поля, использующие пространственную дис- кретизацию моделируемого объекта на мелкой сетке. Наиболее известным и широко используемым, по-видимому, является так называемый метод FDTD $^{1}$ (метод конечной разности во временной области), предложенный предположительно в [4]. Однако, как справедливо отмечается в [5], метод FDTD требует значительных вычислительных ресурсов и потому неконкурентоспособен. Так, например, при решении конкретной задачи [6] с использованием метода ILCM $^{2}$ удалось добиться на 3 десятичных порядка большего быстродействия, чем при использовании методов FDTD и $\mathrm{MOM}^{3}[6]$.

При реализации метода ILCM [7,8] требуется решение интегрального уравнения; метод не приспособлен для решения ряда актуальных задач и может потребовать немалых вычислительных ресурсов. Даже специальная адаптация метода ILCM под некоторые задачи, требующие многократного вычисления поля (например, задачи расчета помехонесущего поля и оценки внутренней электромагнитной совместимости в микросхемах и на печатных платах), не приводит к необходимому и достаточному снижению времени счета и является лишь паллиативом.

Заслуживает особого внимания модифицированный метод эквивалентной схемы частичного элемента (PEEC-method $)^{4}[8,9]$, разработку которого после выхода монографии [8], по-видимому, можно считать в основном завершенной. Метод ориентирован на решение задач электроники для частоты $\omega>0$ и адаптирован для расчетов во временной области. Однако, как отмечается в [10], метод РЕЕС не учитывает излучение и время распространения электромагнитной волны; необходимость анализа структур, размеры которых соизмеримы с длиной волны, потребовала серьезно усложнить метод. Гибридный FDTD/PEEC-метод, предложенный в [10], также

\footnotetext{
${ }^{1}$ FDTD - Finite Difference Time Domain.

2 ILCM - Intermediate Level Circuit Model.

${ }^{3}$ MOM - Method Of Moments.

${ }^{4}$ PEEC - Partial Element Equivalent Circuit.
} 
не обладает достаточным быстродействием, приемлемым при решении ряда проектных задач электроники, и не сможет существенно изменить ситуацию; необходим прагматичный, „технический“ подход к решению задачи, получившей в теоретической физике решение, не всегда пригодное для практического использования. Полученные с помощью вычисления $S$-интегралов для решения задач электроники результаты относятся в основном к оценке взаимовлияния компланарных проводников, параллельных плоским границам раздела слоев; для такой оценки расчет вертикальной компоненты поля не нужен. Взаимовлияние проводов, лежащих во взаимно перпендикулярных плоскостях, не рассматривалось (по сведениям автора). В то же время при оценке взаимовлияния некомпланарных проводников необходимо использовать, как минимум, две компоненты поля, в том числе вертикальную. Дальнейшее развитие методов расчета поля в плоскослоистой среде остается по-прежнему актуальным.

\section{Метод эквивалентной постоянной распространения}

Реальной альтернативой названным методам расчета поля в некоторых случаях может явиться метод эквивалентной постоянной распространения (ЭПР), позволяющий избежать вычисления $S$-интегралов. Метод ЭПР ориентирован приблизительно на тот же частотный диапазон, что и метод РЕЕС, но обладает существенно меньшей вычислительной емкостью. Метод ЭПР был предложен в [11] и затем развит в $[12,13]$ применительно к задаче расчета перекрестных наводок в компланарных проводниках радиоэлектронного модуля. Параметры эквивалентной схемы, которая используется для моделирования таких наводок в системах автоматизированного проектирования (САПР), при использовании метода ЭПР рассчитываются [14] не только через статическую составляющую поля, но в отличие от РЕEС-метода с учетом также и поля излучения.

В [11] была проведена апробация метода ЭПР на ряде задач, для которых известно строгое решение, подтверждена корректность используемого подхода и проведена оценка области корректного использования метода. Там же было показано, что метод ЭПР позволяет свести решение волнового уравнения к решению уравнений Лапласа для электрического и векторного магнитного потенциалов поля. Однако применение описанной в [11] схемы использования метода ЭПР, основанной на использовании математической модели поля в однородном пространстве, для расчета вертикальной компоненты поля невозможно, поскольку компонента поля горизонтального источника, перпендикулярная границам раздела слоев (вертикальная компонента), возникает лишь в слоистой среде и в однородном пространстве отсутствует. Поэтому при расчете вертикальной компоненты математическую модель поля в плоскослоистой среде целесообразно описывать аналитическим выражением, соответствующим двум полупространствам с плоской границей раздела, а многослойность среды учитывать с помощью ЭПР, как предлагается в [11], т. е. используя ЭПР вместо постоянной распространения, определяемой в классической трактовке этого понятия только электрофизическими свойствами материала слоя и частотой. Такая же схема пригодна и для расчета горизонтальной компоненты поля. Решения, получаемые альтернативными методами, обладают большей вычислительной емкостью.

\section{Поле вблизи плоской границы раздела полупространств}

Рассмотрим расчет поля горизонтального источника в плоскослоистой среде более подробно. Для решения многих прикладных задач технической физики электромагнитное поле удобно характеризовать напряженностью электрического поля $E$, которая связана с векторным магнитным $(A)$ и электрическим потенциалами хорошо известным соотношением:

$$
E=-\nabla \varphi+i \omega A, \quad \mathbf{E}=k^{2} \Pi+\overline{\operatorname{grad} \operatorname{div} \Pi} .
$$

Для вычисления потенциалов $A$ и $\varphi$ можно воспользоваться классическими результатами, полученными для поляризационного потенциала поля вблизи плоской границы полупространств. Как показано в [15], в случае элементарного диполя, расположенного на плоской границе раздела полупространств и ориентированного вдоль оси абсцисс (аппликата границы $z_{1}=0$, аппликата диполя $\left.z_{0}=0\right)$, на частоте $\omega \geq 0$ в $j$-м полупространстве $(j=1,2)$ горизонтальная компонента поляризационного потенциала, соответствующая такой структуре, описывается выражениями

$$
\left.\begin{array}{rl}
\Pi_{x_{1}}= & \frac{2}{n^{2}} \int_{0}^{\infty} J_{0}(\lambda r) \Phi_{2,1}(\lambda) d \lambda, \quad z \leq 0, \\
\Pi x_{2}= & \frac{\exp \left(i k_{2} R_{1}\right)}{R_{1}}-\frac{\exp \left(i k_{2} R_{2}\right)}{R_{2}} \\
& +2 \int_{0}^{\infty} J_{0}(\lambda r) \Phi_{2,2}(\lambda) d \lambda, \quad z \geq 0,
\end{array}\right\}
$$

где в общем случае $\Phi_{m, j}$ - определяемая из граничных условий для горизонтальной компоненты математическая модель [16] плоскослоистой среды, в которой рассчитывается поле, причем индекс $m-$ число слоев среды, а $j-$ номер слоя, $k_{j}-$ постоянная распространения электромагнитной энергии в $j$-м слое, $\Phi_{2,1}(\lambda)=\frac{\exp \left(\alpha_{1} z-\alpha_{2} z_{0}\right)}{\alpha_{1}+\alpha_{2}} \lambda, \quad z \leq 0, \quad \Phi_{2,2}(\lambda)=\frac{\exp \left[-\left(z+z_{0}\right) \alpha_{2}\right]}{\alpha_{1}+\alpha_{2}} \lambda$, $z \geq 0 \quad[15], \alpha_{j}=\sqrt{\lambda^{2}-k_{j}^{2}}, j=1,2, \lambda$ - параметр 
разделения [2], именуемый в [17] параметром разложения, $\theta=\arccos x / r-$ азимут, $J_{0}-$ функция Бесселя первого рода нулевого порядка, $n^{2}=k_{1}^{2} / k_{2}^{2}, x_{0}, y_{0}, z_{0}-$ абсцисса, ордината и аппликата источника поля; $x$, $y, z$ - абсцисса, ордината и аппликата точки, где вычисляется поле; радиус $r=\sqrt{\left(x-x_{0}\right)^{2}+\left(y-y_{0}\right)^{2}}$; $R_{1}=\sqrt{r^{2}+\left(z-z_{0}\right)^{2}} ; R_{2}=\sqrt{r^{2}+\left(z+z_{0}\right)^{2}} ; i$ - мнимая единица (мнимое число, удовлетворяющее соотношению $\left.i^{2}=-1\right) ; \pi=3.14159 \ldots$; здесь и далее слои (и полупространства) считаются изотропными, гомогенными, неограниченными в азимутальном направлении и имеющими плоскопараллельные границы раздела, электромагнитные процессы считаются монохроматическими, а электрофизические характеристики слоев частотонезависимыми.

Выражения (2) удовлетворяют волновому уравнению и граничным условиям для плоской границы полупространств [15]

$$
k_{2}^{2} \Pi_{x_{2}}=k_{1}^{2} \Pi_{x_{1}} \quad \text { и } \quad k_{2}^{2} \frac{\partial \Pi_{x_{2}}}{\partial z}=k_{1}^{2} \frac{\partial \Pi_{x_{1}}}{\partial z} .
$$

Вертикальная компонента поляризационного потенциала в рассматриваемой структуре описывается выражениями [15]

$$
\begin{aligned}
& \Pi_{z_{2}}=-\frac{2 \cos \theta}{k_{2}^{2}} \int_{0}^{\infty} J_{1}(\lambda r) \Omega_{2,1}(\lambda) \lambda^{2} d \lambda, \quad z \leq 0 \\
& \Pi_{z_{1}}=-\frac{2 \cos \theta}{k_{1}^{2}} \int_{0}^{\infty} J_{1}(\lambda r) \Omega_{2,2}(\lambda) \lambda^{2} d \lambda, \quad z \geq 0,
\end{aligned}
$$

где $\Omega_{2,1}(\lambda)=\frac{\alpha_{2}-\alpha_{1}}{n^{2} \alpha_{2}+\alpha_{1}} \exp \left(\alpha_{1} z-\alpha_{2} z_{0}\right), \quad z \leq 0-$ математическая модель структуры, состоящей из полупространств с плоской границей раздела для $z$-компоненты [15]; $\Omega_{2,2}(\lambda)=\frac{\alpha_{2}-\alpha_{1}}{n^{2} \alpha_{2}+\alpha_{1}} \exp \left[-\alpha_{2}\left(z+z_{0}\right)\right]-$ то же для $z \geq 0[15] ; J_{1}-$ функция Бесселя первого рода первого порядка.

При выводе формул (4) и (5) использованы граничные условия [15]

$$
k_{2}^{2} \Pi_{z_{2}}=k_{1}^{2} \Pi_{z_{1}} \quad \text { и } \quad \frac{\partial \Pi_{z_{2}}}{\partial z}-\frac{\partial \Pi_{z_{1}}}{\partial z}=\frac{\partial \Pi_{x_{1}}}{\partial x}-\frac{\partial \Pi_{x_{2}}}{\partial x} .
$$

\section{Получение математических моделей поля для малых расстояний}

Как отмечено выше, имеется настоятельная необходимость снижения вычислительной емкости решения рассматриваемой задачи, поэтому выражения (1) и (2) следует упростить с помощью надлежащей аппроксимации. Для этого обычно вводятся (см., например, $[1,17,18]$ ) ограничения на значения постоянных распространения и параметра разделения, которые, возможно, корректны при математическом моделировании распространения радиоволн, при решении задач геологии, молниезащиты, но совершенно нехарактерны для материалов и расстояний микроэлектроники. Более того, физический смысл вводимых формально допущений иногда вообще неуловим.

В [19] было теоретически показано, что в квазистатике погрешность аппроксимации функций $\Phi_{m, j}(\lambda)$ на малых $\lambda$ сказывается на значении функции Грина в основном на больших расстояниях, а для расчета поля на малых расстояниях важно повышать точность аппроксимации математической модели слоистой среды в области больших значений $\lambda$ и при $\lambda=\infty$. В [20] эти выводы были подтверждены средствами вычислительного эксперимента и отмечено, что для функций Грина в области, где эти функции являются гладкими функциями частоты (каковыми они и являются, моделируя отсутствие объемных резонансных явлений), эти выводы справедливы в области нижних частот, где объемные резонансы в микроэлектронных конструктивах заведомо отсутствуют.

Учитывая прикладной характер решаемой задачи технической физики (ориентация на микроэлектронику), выражения (2) и (4), (5) целесообразно упростить, преобразовав их для случая малых расстояний. Такую возможность дают результаты работ $[19,20]$. Тогда для больших значений $\lambda \gg\left|k_{j}\right|$, соответствующих малым расстояниям (и/или области низких частот), $\alpha_{j} \approx \lambda$ и формулы (2) для элементарного диполя, расположенного на плоской границе раздела полупространств, примут вид

$$
\begin{aligned}
\Pi_{x_{1}} & =\frac{1}{n^{2}} \int_{0}^{\infty} J_{0}(\lambda r) \exp \left[\lambda\left(z-z_{0}\right)\right] d \lambda, \quad z \leq 0, \\
\Pi_{x_{2}} & =\frac{\exp \left(i k_{2} R_{1}\right)}{R_{1}}-\frac{\exp \left(i k_{2} R_{2}\right)}{R_{2}} \\
& +\int_{0}^{\infty} J_{0}(\lambda r) \exp \left[-\lambda\left(z+z_{0}\right)\right] d \lambda, \quad z \geq 0,
\end{aligned}
$$

или с учетом тождества Вебера-Липшица [21]

$$
\begin{gathered}
\Pi_{x_{1}}=\frac{1}{n^{2} R_{1}}, \quad z \leq 0, \\
\Pi_{x_{2}}=\frac{\exp \left(i k_{2} R_{1}\right)}{R_{1}}-\frac{\exp \left(i k_{2} R_{2}\right)}{R_{2}}+\frac{1}{R_{2}}, \quad z \geq 0 .
\end{gathered}
$$

Используя приближенное равенство $\alpha_{j} \approx \lambda[1-$ $\left.-k_{j}^{2} /\left(2 \lambda^{2}\right)\right]$, выражения (4) и (5) для вертикальной компоненты можно представить в виде

$$
\begin{aligned}
\Pi_{z_{1}} & =\frac{\left(k_{2}^{2}-k_{1}^{2}\right) \cos \theta}{k_{2}^{2}\left(n^{2}+1\right)} \int_{0}^{\infty} J_{1}(\lambda r) \exp \left[\lambda\left(z-z_{0}\right)\right] d \lambda, \\
& z-z_{0} \leq 0
\end{aligned}
$$




$$
\begin{aligned}
\Pi_{z_{2}} & =\frac{\left(k_{2}^{2}-k_{1}^{2}\right) \cos \theta}{k_{1}^{2}\left(n^{2}+1\right)} \int_{0}^{\infty} J_{1}(\lambda r) \exp \left[-\lambda\left(z+z_{0}\right)\right] d \lambda, \\
& z+z_{0} \geq 0 .
\end{aligned}
$$

Взяв интегралы в выражениях (9) и (10), воспользовавшись равенством $\cos \theta=x / r$, получим

$$
\begin{aligned}
& \Pi_{z_{1}}=\frac{\left(k_{2}^{2}-k_{1}^{2}\right) x}{k_{2}^{2}\left(n^{2}+1\right) r^{2}}\left(1-\frac{z-z_{0}}{R_{1}}\right), \quad z-z_{0} \leq 0, \\
& \Pi_{z_{2}}=\frac{\left(k_{2}^{2}-k_{1}^{2}\right) x}{k_{1}^{2}\left(n^{2}+1\right) r^{2}}\left(1-\frac{z+z_{0}}{R_{2}}\right), \quad z+z_{0} \geq 0 .
\end{aligned}
$$

Принятая для получения выражений (7), (8), (11), (12) аппроксимация обеспечивает наибольшую точность при больших $\lambda \gg\left|k_{j}\right|$ (т.е. на малых расстояниях $[19,20]$ ) как на низких, так и на высоких частотах, и при $\lambda=\infty$ ошибка такой аппроксимации равна нулю на любой конечной частоте. Таким образом, выражения (7), (8), (11) и (12) дают решение волнового уравнения (строго говоря, приближенное) относительно поляризационного потенциала поля элементарного горизонтального диполя у плоской границы (и на границе) полупространств для малых расстояний $r$ на нижних (не значит низких!) частотах.

Для расчета поля в многослойной среде можно использовать формулы (11) и (12) для полупространств; многослойность среды учитывается методом ЭПР.

\section{Расчет ЭПР}

В многослойной среде (при $m>2) k_{e}-$ это ЭПР, вычисляемая с учетом влияния всех слоев среды по формуле

$$
k_{e}(r, z)=\sqrt{\varepsilon_{0} \varepsilon_{e} \mu_{0} \mu_{e} \omega^{2}+i \mu_{0} \mu_{e} \sigma_{e} \omega},
$$

где $\sigma_{e}-$ „кажущаяся““ активная проводимость слоистой среды с учетом влияния всех слоев; $\varepsilon_{e}$ и $\mu_{e}-$ эквивалентные относительные диэлектрическая и магнитная проницаемости слоистой среды; константа Кулона $\varepsilon_{0}=10^{-9} /(36 \pi) \mathrm{F} / \mathrm{m} ; \mu_{0}=4 \pi \cdot 10^{-7} \mathrm{H} / \mathrm{m}$ - константа Био-Савара; $\omega-$ угловая частота; значения $\sigma_{e}, \varepsilon_{e}$ и $\mu_{e}$ рассчитываются при $\omega=0$, т.е. на основе решения уравнений Лапласа; нижний индекс $e$ при обозначении величин здесь и ниже указывает, что величина является эквивалентной, т.е. рассчитывается с учетом слоистости среды.

Методы расчета эквивалентной („кажущейся“) активной проводимости на постоянном токе хорошо известны в геологии. Известны как прямые методы расчета „кажущейся“ активной проводимости для изотропных и анизотропных [22] слоистых сред, так и косвенные, основанные на идее использования теории подобия, как предлагается в [23].
Значения эквивалентных относительных проницаемостей $\varepsilon_{e}$ и $\mu_{e}$ как для горизонтальной, так и для вертикальной компонент поля рассчитываются при $\omega=0$ как отношение соответствующих функций Грина для многослойной среды и среды, представляющей собой полупространства с плоской границей раздела, причем электрофизические характеристики $\sigma_{1}, \varepsilon_{1}$ и $\mu_{1}$ нижнего полупространства совпадают с характеристиками подстилающего слоя многослойной среды, а характеристики $\sigma_{2}, \varepsilon_{2}$ и $\mu_{2}$ верхнего полупространства совпадают с характеристиками накрывающего слоя, в котором находится элементарный диполь. Расчет $\sigma_{e}, \varepsilon_{e}$ и $\mu_{e}$ не требует вычисления $S$-интегралов, а предполагает вместо этого решение уравнений Лапласа для вектора Герца или для электрического $\varphi$ и векторного магнитного А-потенциалов. Такое решение требует формулировать граничные условия при $\omega=0$ на границах раздела слоев с проводимостями $\sigma_{j}$ и относительными диэлектрическими $\varepsilon_{j}$ и магнитными $\mu_{j}$ проницаемостями $(j=\overline{1, m})$, а также найти математические модели слоистой среды $[16,20]$ (т.е. функции $\Phi_{m, j}, \Omega_{m, j}$ для векторного потенциала и функции $\Lambda_{m, j}$ для электрического) при $\omega=0$. В силу известного соотношения $\mathbf{A}=\mu(-i \omega \varepsilon+\sigma) \Pi[17]$ векторный магнитный $\mathbf{A}$ и поляризационный П потенциалы связаны между собой коэффициентом пропорциональности, значение которого в пределах каждого гомогенного слоя от координат не зависит и изменяется только при переходе через границу раздела слоев, поэтому граничные условия для магнитного потенциала могут быть сформированы на основе представленных выше граничных условий (3) и (6) для вектора Герца.

При $\omega=0$ условия (3) и (6) на плоских границах раздела слоев с аппликатами $z=z_{j}$ приводятся к виду $(1 \leq j \leq m)$ :

$$
\begin{gathered}
A_{x, j+1}=A_{x, j} \quad \text { и } \quad \frac{\partial A_{x, j+1}}{\partial z}=\frac{\partial A_{x, j}}{\partial z} . \\
A_{z, j+1}=A_{z, j} ; \quad \frac{1}{\mu_{j+1} \varepsilon_{j+1}} \frac{\partial A_{z, j+1}}{\partial z}-\frac{1}{\mu_{j} \varepsilon_{j}} \frac{\partial A_{z, j}}{\partial z} \\
=\frac{1}{\mu_{j} \varepsilon_{j}} \frac{\partial A_{x, j}}{\partial x}-\frac{1}{\mu_{j+1} \varepsilon_{j+1}} \frac{\partial A_{x, j+1}}{\partial x} .
\end{gathered}
$$

В пределах идеально проводящих слоев и на их границах потенциалы $A$ и $\varphi$ равны нулю. Для $x$-компоненты в случае непроводящих немагнитных сред, имеющих общую плоскую границу при $z=z_{j}$, на частоте $\omega \geq 0$ используются условия (13), а для $z$-компоненты условия (14), которые можно представить в виде

$$
\begin{aligned}
A_{z, j+1}=A_{z, j} \quad \text { и } & \frac{1}{\varepsilon_{j+1}} \frac{\partial A_{z, j+1}}{\partial z}-\frac{1}{\varepsilon_{j}} \frac{\partial A_{z, j}}{\partial z} \\
& =\frac{1}{\varepsilon_{j}} \frac{\partial A_{x, j}}{\partial x}-\frac{1}{\varepsilon_{j+1}} \frac{\partial A_{x, j+1}}{\partial x} .
\end{aligned}
$$


Для электрического потенциала граничные условия [23]

$$
\varphi_{j+1}=\varphi_{j} \quad \text { и } \quad \varepsilon_{j+1} \frac{\partial \varphi_{j+1}}{\partial z}=\varepsilon_{j} \frac{\partial \varphi_{j}}{\partial z} .
$$

Будем теперь считать, что источник поля (элементарный диполь) расположен в $\vartheta+1-м$ слое над границей с аппликатой $z=z_{\vartheta}=0$ или на этой границе. ${ }^{5}$

Для горизонтальной компоненты векторного магнитного потенциала в $j$-м слое $m$-слойной среды $(1 \leq j \leq \vartheta)$ под верхней границей $\vartheta$-го слоя эквивалентная относительная магнитная проницаемость, по определению:

$$
\begin{gathered}
\mu_{e, x, j}=\frac{A_{x, j}}{A_{2,1}}=\int_{0}^{\infty} J_{0}(\lambda r) \Phi_{m, j}(\lambda) d \lambda / \int_{0}^{\infty} J_{0}(\lambda r) \Phi_{2,1}(\lambda) d \lambda, \\
z \leq z_{j} \leq z_{\vartheta},
\end{gathered}
$$

где $\Phi_{m, j}-$ математическая модель $m$-слойной среды для $j$-го слоя, в котором вычисляется поле, получаемая из граничных условий (13) при $\omega=0$; интеграл в знаменателе с точностью до постоянного коэффициента описывает $x$-компоненту поля элементарного диполя в нижнем полупространстве.

То же в $j$-м слое над верхней границей $\vartheta$-го слоя $(m \geq j \geq \vartheta)$

$$
\begin{gathered}
\mu_{e, z, j}=\frac{A_{x, j}}{A_{2,2}}=\int_{0}^{\infty} J_{0}(\lambda r) \Phi_{m, j}(\lambda) d \lambda / \int_{0}^{\infty} J_{0}(\lambda r) \Phi_{2,2}(\lambda) d \lambda \\
z \geq z_{j} \geq z_{\vartheta},
\end{gathered}
$$

где интеграл в знаменателе с точностью до постоянного коэффициента описывает $x$-компоненту поля элементарного диполя в верхнем полупространстве.

То же для вертикальной компоненты поля в $j$-м слое в области $z \leq z_{j} \leq z_{\vartheta}$ :

$$
\mu_{e, z, j}=\frac{A_{z, j}}{A_{2,1}}=\int_{0}^{\infty} J_{1}(\lambda r) \Omega_{m, j}(\lambda) d \lambda / \int_{0}^{\infty} J_{1}(\lambda r) \Omega_{2,1}(\lambda) d \lambda .
$$

Для вертикальной компоненты в $j$-м слое в области $z \geq z_{j} \geq z_{\vartheta}$

$$
\mu_{e, z, j}=\frac{A_{z, \vartheta}}{A_{2,2}}=\int_{0}^{\infty} J_{1}(\lambda r) \Omega_{m, \vartheta}(\lambda) d \lambda / \int_{0}^{\infty} J_{1}(\lambda r) \Omega_{2,2}(\lambda) d \lambda .
$$

Для вычисления эквивалентной относительной диэлектрической проницаемости $\varepsilon_{e}$ функцию Грина в слоистой среде ищем, используя граничные условия (16)

\footnotetext{
5 Для диполя на границе раздела при формировании системы уравнений, используя различные граничные условия, полагаем, что диполь отделен от границы весьма тонким зазором, свободным от источников поля. Для нахождения функций $\Phi, \Omega, \Lambda$ толщина зазора устремляется к нулю и осуществляется переход к пределу.
}

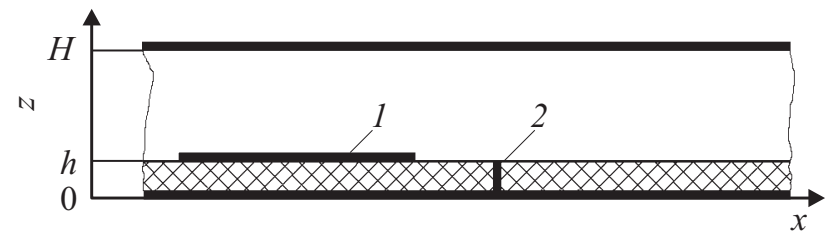

Четырехслойная среда, адекватная проводнику на плате с верхним и нижним экранами: 1 - проводник-источник поля, 2 - заземляющая перемычка (проводник-рецептор).

для электрического потенциала $\varphi$ на частоте $\omega=0$. По определению эквивалентной диэлектрической проницаемости для поля в $j$-м слое $(1 \leq \vartheta \leq j)$ по аналогии c [11]:

$$
\varepsilon_{e, j}=\frac{\varphi_{2}}{\varphi_{j}}=\int_{0}^{\infty} J_{0}(\lambda r) \Lambda_{2,2}(\lambda) d \lambda / \int_{0}^{\infty} J_{0}(\lambda r) \Lambda_{m, j}(\lambda) d \lambda,
$$

$z \geq z_{j} \geq z_{\vartheta}$

То же для $1 \leq j \leq \vartheta$ :

$$
\begin{aligned}
& \varepsilon_{e, j}=\frac{\varphi_{1}}{\varphi_{j}}=\int_{0}^{\infty} J_{0}(\lambda r) \Lambda_{2,1}(\lambda) d \lambda / \int_{0}^{\infty} J_{0}(\lambda r) \Lambda_{m, j}(\lambda) d \lambda \\
& z \leq z_{j} \leq z_{\vartheta}
\end{aligned}
$$

По найденным значениям эквивалентных проницаемостей находятся значения ЭПР, которые используются для расчета поляризационного потенциала по формулам (7), (8), (11), (12). Вычислив затем потенциалы по формулам $\mathbf{A}=\mu_{e}\left(-i \omega \varepsilon_{e}+\sigma_{e}\right) \Pi$ и $\varphi=-\operatorname{div} \Pi$, находим по формуле (1) напряженность электрического поля единичного элементарного диполя, значений которой достаточно для решения широкого класса задач.

\section{Приложение}

\section{Пример использования методики}

Рассмотрим расчет ЭДС помехи, наведенной в вертикальной заземляющей перемычке на металлизированной диэлектрической печатной плате горизонтальным прямоугольным пленочным проводником. Верхний экран образован металлизированным слоем соседней („верхней“') платы. Соответствующая конструкция схематично представлена на рисунке. Диэлектрики с диэлектрическими проницаемостями $\varepsilon_{3}$ (для пространства над платой) и $\varepsilon_{2}$ (для платы) полагаем непроводящими и немагнитными, экраны полагаем идеально проводящими. Источник поля (проводник прямоугольного сечения шириной $W$, длиной $l$, толщиной $t$ и характеристическим сопротивлением $114 \Omega$ ) ориентирован вдоль оси абсцисс. 
Для расчета поля методом ЭПР необходимо определить эквивалентные проницаемости. Для определения эквивалентной диэлектрической проницаемости требуется знать потенциал электрического поля в квазистационарном приближении для каждого слоя среды, адекватной реальному конструктиву. Для описания электрического потенциала поля в слое над платой (см. рисунок) будем использовать выражение $\int_{0}^{\infty} J_{0}(\lambda r) \Lambda_{4,3}(\lambda) d \lambda$, которое включает математическую модель $\Lambda_{4,3}\left(\lambda, z, z_{0}\right)=$ $=\exp \left(-\lambda\left|z-z_{0}\right|\right)+s_{3}(\lambda) \exp \left[\lambda\left(z-z_{0}\right)+p_{3}(\lambda) \exp [\lambda(x-z)]\right.$, а для слоя, который соответствует плате, будем использовать модель $\Lambda_{4,2}\left(\lambda, z, z_{0}\right)=s_{2}(\lambda) \exp \left[\lambda\left(z-z_{0}\right)\right]+$ $+p_{2}(\lambda) \exp [\lambda(x-z)]$. Записав с помощью этих выражений граничные условия (16), получим систему интегральных уравнений относительно неизвестных $s_{v}(\lambda)$ и $p_{v}(\lambda)(v=1,2)$; число уравнений необходимо и достаточно для отыскания неизвестных функций $s_{v}$ и $p_{v}$. С помощью интеграла Фурье-Бесселя [24] переходим к системе линейных алгебраических уравнений и тогда граничные условия примут вид:

$$
\begin{gathered}
s_{3}(\lambda) \exp \left[\lambda\left(H-z_{0}\right)\right]+p_{3}(\lambda) \exp \left[\lambda\left(z_{0}-H\right)\right] \\
+\exp \left(\lambda\left|H-z_{0}\right|\right)=0, \\
s_{3}(\lambda) \exp \left[\lambda\left(h-z_{0}\right)\right]+p_{3}(\lambda) \exp \left[\lambda\left(z_{0}-h\right)\right] \\
+\exp \left|\lambda\left(h-z_{0}\right)\right|=s_{2}(\lambda) \exp \left[\lambda\left(h-z_{0}\right)\right] \\
+p_{2}(\lambda) \exp \left[\lambda\left(z_{0}-h\right)\right], \\
s_{2}(\lambda) \exp \left(-\lambda z_{0}\right)+p_{2}(\lambda) \exp \left(\lambda z_{0}\right)=0, \\
\varepsilon_{3}\left\{s_{3}(\lambda) \exp \left[\lambda\left(h-z_{0}\right)\right]-p_{3}(\lambda) \exp \left[\lambda\left(z_{0}-h\right)\right]\right. \\
\left.+\exp \left|-\lambda\left(h-z_{0}\right)\right|\right\}=\varepsilon_{2}\left\{s_{2}(\lambda) \exp \left[\lambda\left(h-z_{0}\right)\right]\right. \\
\left.-p_{2}(\lambda) \exp \left[\lambda\left(z_{0}-h\right)\right]\right\} .
\end{gathered}
$$

Решив эту систему уравнений, получим, что

$$
s_{3}=\frac{2 \varepsilon_{3}}{\left(\varepsilon_{2} D-\varepsilon_{3}\right)\left\{\exp \left[2 \lambda\left(h-z_{0}\right)\right]+\exp (-2 H)\right\}},
$$

где

$$
\begin{gathered}
D=\frac{1+\exp (-2 h)}{1-\exp (-2 h)} \frac{1-\exp [2 \lambda(H-h)]}{1+\exp [2 \lambda(H-h)]}, \\
p_{2}=-s_{3} \frac{1-\exp [2 \lambda(H-h)]}{1+\exp [2 \lambda(H-h)]}, \\
p_{3}=-s_{3} \exp \left[2 \lambda\left(H-z_{0}\right)\right]-1, \\
s_{2}=-p_{2} \exp \left(-2 \lambda z_{0}\right) .
\end{gathered}
$$

Математическая модель полупространств с плоской границей для расчета $\varepsilon_{e, v}(v=1,2)$ находится аналогично. Для этого следует рассмотреть при $\omega=0$ электрический потенциал в структуре, состоящей из полупространств, верхнее из которых имеет свойства свободного пространства, а нижнее - свойства материала платы. Потенциал в верхнем полупространстве описывается с помощью математической модели
$\Lambda_{2,2}\left(\lambda, z, z_{0}\right)=\exp \left(-\lambda\left|z-z_{0}\right|\right)+q_{2}(\lambda) \exp \left[\lambda\left(z_{0}-z\right)\right], \quad \mathrm{a}$ в нижнем полупространстве - с помощью математической модели $\Lambda_{2,1}\left(\lambda, z, z_{0}\right)=g_{1}(\lambda) \exp \left[\lambda\left(z-z_{0}\right)\right]$. Граничные условия (16) вкупе с интегралом Фурье-Бесселя дают систему, решение которой имеет вид

$$
\begin{gathered}
q_{2}(\lambda)=\frac{\varepsilon_{3} \exp \left[-2 \lambda\left(z_{0}-h\right)\right]}{\varepsilon_{2}+\varepsilon_{3}}, \\
g_{1}(\lambda)=\frac{\varepsilon_{3}-\varepsilon_{2} \exp \left[2 \lambda\left(z_{0}-h\right)\right]}{\varepsilon_{3}+\varepsilon_{2}}+1 .
\end{gathered}
$$

Тогда эквивалентная относительная диэлектрическая проницаемость $(j=3)$

$$
\varepsilon_{e, 3}=\frac{\int_{0}^{\infty} J_{0}(\lambda r)\left\{\exp \left(-\lambda\left|z-z_{0}\right|\right)+s_{3}(\lambda) \exp \left[\lambda\left(z-z_{0}\right)\right]+\right.}{\left.+p_{3}(\lambda) \exp \left[\lambda\left(z-z_{0}\right)\right]\right\} d \lambda} .
$$

То же для слоя с номером $j=2$, который соответствует плате

$$
\varepsilon_{e, 2}=\frac{\int_{0}^{\infty} J_{0}(\lambda r)\left\{s_{2}(\lambda) \exp \left[\lambda\left(z-z_{0}\right)\right]+\right.}{\left.+p_{2}(\lambda) \exp \left[\lambda\left(z_{0}-z\right)\right]\right\} d \lambda} .
$$

Формирование математической модели четырехслойной структуры, соответствующей конструктиву на рисунке, для $x$-компоненты векторного потенциала при расчете эквивалентной относительной магнитной проницаемости $\mu_{e}$ проводится аналогично, но на основе использования граничных условий (13). Решение соответствующей системы уравнений показывает, что $x$-компонента при $\omega=0$ для слоя над платой описывается с помощью функции

$$
\begin{aligned}
& \Phi_{4,3}\left(\lambda, z, z_{0}\right)=u_{3}(\lambda) \exp \left[\lambda\left(z_{0}-z\right)\right] \\
& \quad+w_{3}(\lambda) \exp \left[\lambda\left(z-z_{0}\right)\right]+\exp \left(-\lambda\left|z-z_{0}\right|\right),
\end{aligned}
$$

где

$$
\begin{gathered}
w_{3}(\lambda)=\frac{2}{(D-1)\left\{\exp \left[2 \lambda\left(h-z_{0}\right)\right]+\exp (-2 \lambda H)\right\}}, \\
D=\frac{1+\exp (-2 h)}{1-\exp (-2 h)} \frac{1-\exp [2 \lambda(H-h)]}{1+\exp [2 \lambda(H-h)]} \\
u_{3}(\lambda)=-w_{3}(\lambda) \exp \left[2 \lambda\left(H-z_{0}\right)\right]-1 .
\end{gathered}
$$

Для слоя, соответствующего плате, $x$-компонента описывается с помощью функции

$\Phi_{4,2}\left(\lambda, z, z_{0}\right)=u_{2}(\lambda) \exp \left[\lambda\left(z_{0}-z\right)\right]+w_{2}(\lambda) \exp \left[\lambda\left(z-z_{0}\right)\right]$,

где

$$
\begin{gathered}
u_{2}(\lambda)=-w_{3} \frac{1-\exp [2 \lambda(H-h)]}{1+\exp [2 \lambda(H-h)]}, \\
w_{2}=-u_{2}(\lambda) \exp \left(-2 \lambda z_{0}\right) .
\end{gathered}
$$


Чтобы получить аналитические выражения для эквивалентных относительных магнитных проницаемостей, соответствующих каждой из компонент векторного потенциала, следует еще рассмотреть этот потенциал в структуре из полупространств, верхнее из которых имеет свойства свободного пространства, а нижнее - свойства материала платы и соответствующие нижнему полупространству функции $\Omega_{2,1}$ и $\Phi_{2,1}$. Для $x$-компоненты эквивалентная проницаемость $\mu_{e}$ в верхнем полупространстве описывается формулой (18) с помощью математической модели $\Phi_{2,2}\left(\lambda, z, z_{0}\right)=q_{2}(\lambda) \exp \left[\lambda\left(z_{0}-z\right)\right]+\exp \left(-\lambda\left|z-z_{0}\right|\right), \quad$ a в нижнем - с помощью математической модели $\Phi_{2,1}\left(\lambda, z, z_{0}\right)=g_{1}(\lambda) \exp \left[\lambda\left(z-z_{0}\right)\right]$. Граничные условия (15) вкупе с интегралом Фурье-Бесселя дают систему, решение которой имеет вид

$$
q_{2}(\lambda)=\frac{\exp \left[-2 \lambda\left(z_{0}-h\right)\right]}{2}, g_{1}(\lambda)=\frac{3-\exp \left[2 \lambda\left(z_{0}-h\right)\right]}{2} .
$$

Для $x$-компоненты векторного потенциала в объеме платы по формуле (17)

$$
\mu_{e, x, 2}=\frac{\int_{0}^{\infty} J_{0}(\lambda r)\left\{w_{2}(\lambda) \exp \left[\lambda\left(z-z_{0}\right)\right]+\right.}{\int_{0}^{\infty} J_{0}(\lambda r)\left\{g_{1}(\lambda) \exp \left[\lambda\left(z-z_{0}\right)\right]\right\} d \lambda},
$$

то же для слоя над платой

$$
\mu_{e, x, 3}=\frac{\int_{0}^{\infty} J_{0}(\lambda r)\left\{w_{3}(\lambda) \exp \left[\lambda\left(z-z_{0}\right)\right]+\right.}{\left.+u_{3}(\lambda) \exp \left[\lambda\left(z_{0}-z\right)\right]\right\} d \lambda} .
$$

Для z-компоненты векторного потенциала при расчете эквивалентной магнитной проницаемости $\mu_{e}$ по формуле (19) требуется использовать математическую модель $\Omega_{m, j}$ того же типа, что и $\Phi_{m, j}$ для $x$-компоненты, но сформированную на основе граничных условий (15) [15], в которых участвуют обе компоненты векторного потенциала. В любом из четырех ( $j$-м) слоев модели $z$-компонента векторного потенциала описывается при помощи функции вида

$$
A_{z, j}=\int_{0}^{\infty} J_{1}(\lambda r) \Omega_{4, j}\left(\lambda, z, z_{0}\right) d \lambda, \quad j=\overline{1,4} .
$$

Математические модели описываются следующими выражениями:

$$
\begin{gathered}
\Omega_{4,1}=\Omega_{4,4}=0 \\
\Omega_{4,2}\left(\lambda, z, z_{0}\right)=c(\lambda) \exp \left[\lambda\left(z-z_{0}\right)\right]+a(\lambda) \exp \left[\lambda\left(z_{0}-z\right)\right] \\
\Omega_{4,3}\left(\lambda, z, z_{0}\right)=e(\lambda) \exp \left(-\lambda\left|z-z_{0}\right|\right)+f(\lambda) \exp \left[\lambda\left(z_{0}-z\right)\right] .
\end{gathered}
$$

Для четырехслойной структуры граничные условия (15) образуют систему

$$
\begin{gathered}
c(\lambda) \exp \left[\lambda\left(h-z_{0}\right)\right]+a(\lambda) \exp \left[\lambda\left(z_{0}-h\right)\right] \\
=\exp \left(-\lambda\left|h-z_{0}\right|\right)+e(\lambda) \exp \left[\lambda\left(h-z_{0}\right)\right] \\
+f(\lambda) \exp \left[\lambda\left(z_{0}-h\right)\right], \\
\left\{e(\lambda) \exp \left[\lambda\left(h-z_{0}\right)\right]-f(\lambda) \exp \left[\lambda\left(z_{0}-h\right)\right]\right. \\
\left.+\exp \left(-\lambda\left|h-z_{0}\right|\right)\right\} / \varepsilon_{3}-\left\{c(\lambda) \exp \left[\lambda\left(h-z_{0}\right)\right]\right. \\
\left.-a(\lambda) \exp \left[\lambda\left(z_{0}-h\right)\right]\right\} / \varepsilon_{2}=\frac{x}{r} \Gamma, \\
\exp \left(-\lambda\left|H-z_{0}\right|\right)+e(\lambda) \exp \left[\lambda\left(H-z_{0}\right)\right] \\
\quad+f(\lambda) \exp \left[\lambda\left(z_{0}-H\right)\right]=0, \\
c(\lambda) \exp \left(-\lambda z_{0}\right)+a(\lambda) \exp \left(\lambda z_{0}\right)=0,
\end{gathered}
$$

где

$$
\Gamma=-\Phi_{4,2}(\lambda, h) / \varepsilon_{2}+\Phi_{4,3}(\lambda, h) / \varepsilon_{3},
$$

$\Phi_{4, j}$ и $\Omega_{4, j}$ от $x$ не зависят и для $x$-компоненты описываются формулами (П2) и (П1), а для $z$-компоненты формулами (П3) и (П4).

При $z_{0}=h$ решение системы имеет вид

$$
\begin{array}{r}
f(\lambda)=\frac{\frac{x}{r} \Gamma+\{\exp [2 \lambda(h-H)]-1\}\left\{1 / \varepsilon_{3}-1 / \varepsilon_{2}\right\}+\xi}{-\{1+\exp [2 \lambda(h-H)]\} / \varepsilon_{3}-}, \\
-\{1-\exp [2 \lambda(h-H)]\} / \varepsilon_{2}-\xi
\end{array}
$$

где

$$
\begin{gathered}
\xi=2 \frac{\exp [2 \lambda(h-H)]-1}{[\exp (2 \lambda h)-1] \varepsilon_{2}}, \\
e(\lambda)=-[1+f(\lambda)] \exp [2 \lambda(h-H)], \\
a(\lambda)=[1+f(\lambda)] \frac{\exp [2 \lambda(h-H)]-1}{\exp (2 \lambda h)-1}, \\
c(\lambda)=1+f(\lambda)+e(\lambda)-a(\lambda) .
\end{gathered}
$$

Для расчета $m_{e, z, 2}$ требуется еще найти векторный потенциал магнитного поля в структуре из полупространств, верхнее из которых имеет свойства свободного пространства, а нижнее - свойства материала платы. Потенциал в верхнем полупространстве описывается с помощью математической модели $\Omega_{2,2}\left(\lambda, z, z_{0}\right)=\exp \left(-\lambda\left|z-z_{0}\right|\right)+b(\lambda) \exp \left[\lambda\left(z_{0}-z\right)\right]$, a в нижнем - c помощью математической модели $\Omega_{2,1}\left(\lambda, z, z_{0}\right)=d(\lambda) \exp \left[\lambda\left(z-z_{0}\right)\right]$. Граничные условия (15) вкупе с интегралом Фурье-Бесселя дают систему из двух алгебраических уравнений:

$d(\lambda) \exp \left[\lambda\left(h-z_{0}\right)\right]=\exp \left(-\lambda\left|h-z_{0}\right|\right)+b(\lambda) \exp \left[\lambda\left(z_{0}-h\right)\right]$,

$$
\begin{aligned}
\{-b(\lambda) & \left.\exp \left[\lambda\left(z_{0}-h\right)\right]+\exp \left(-\lambda\left|h-z_{0}\right|\right)\right\} / \varepsilon_{3} \\
& +d(\lambda) \exp \left[\lambda\left(z_{0}-h\right)\right] / \varepsilon_{2} \\
& =\frac{x}{r}\left[-\Phi_{2,1}(\lambda, h) / \varepsilon_{2}+\Phi_{2,2}(\lambda, h) / \varepsilon_{3}\right],
\end{aligned}
$$


решение которой имеет вид

$$
b(\lambda)=\frac{\left\{\varepsilon_{3} \varepsilon_{2} \beta_{2} x / r-\varepsilon_{2} \exp \left[\lambda\left(h-z_{0}\right)\right]-\right.}{\left.-\varepsilon_{3} \exp \left[\lambda\left(z_{0}-h\right)\right]\right\} \exp \left[\lambda\left(h-z_{0}\right)\right]},
$$

где

$$
\begin{gathered}
\beta_{2}=\Phi_{21} / \varepsilon_{2}+\Phi_{22} / \varepsilon_{3}, \\
d(\lambda)=1+b(\lambda) \exp \left[2 \lambda\left(z_{0}-h\right)\right] .
\end{gathered}
$$

Тогда для $z$-компоненты векторного потенциала в объеме платы по формуле (19):

$$
\mu_{e, z, 2}=\frac{\int_{0}^{\infty} J_{1}(\lambda r)\left\{c(\lambda) \exp \left[\lambda\left(z-z_{0}\right)\right]+\right.}{\left.+a(\lambda) \exp \left[\lambda\left(z_{0}-z\right)\right]\right\} d \lambda}
$$

То же в слое над платой по формуле (20):

$$
\begin{gathered}
\int_{0}^{\infty} J_{1}(\lambda r)\left\{\exp \left(-\lambda\left|z-z_{0}\right|\right)+\right. \\
\mu_{e, z, 3}=\frac{\left.+e(\lambda) \exp \left[\lambda\left(z-z_{0}\right)\right]+f(\lambda) \exp \left[\lambda\left(z_{0}-z\right)\right]\right\} d \lambda}{\int_{0}^{\infty} J_{1}(\lambda r)\left\{\exp \left(-\lambda\left|z-z_{0}\right|\right)+\right.} \\
\left.+b(\lambda) \exp \left[\lambda\left(z_{0}-z\right)\right]\right\} d(\lambda)
\end{gathered}
$$

\section{Вычисление напряжения помехи}

Как показано выше, расчет ЭПР требует вычисления интегралов двух типов:

$$
\begin{aligned}
I_{1} & =\int_{0}^{\infty} J_{0}(\lambda r) \Phi_{=}\left(\lambda, z-z_{0}\right) d \lambda \\
\text { и } I_{2} & =\int_{0}^{\infty} J_{1}(\lambda r) \Phi_{\perp}\left(\lambda, z-z_{0}\right) d \lambda,
\end{aligned}
$$

где $\Phi_{=}$- математическая модель плоскослоистой среды для электрического потенциала или для горизонтальной составляющей векторного потенциала, $\Phi_{\perp}-$ то же для вертикальной составляющей векторного потенциала.

Обобщая полученные к настоящему времени результаты, можно отметить, что для нетривиальных случаев известные способы вычисления интеграла $I_{1}$ базируются либо на использовании свойств тета-функции [25], либо на аппроксимации функции $\Phi_{=}(\lambda)$ отрезком экспоненциального ряда на интервале $[0, \infty]$ и использовании тождества Вебера-Липшица

$$
\int_{0}^{\infty} J_{0}(\lambda r) \exp \left(-\lambda \tau_{0}\right) d \lambda=\frac{1}{\sqrt{r^{2}+\tau_{0}^{2}}} \quad\left(\tau_{0} \geq 0\right),
$$

где $\tau_{0}-$ масштабирующий множитель $[20,21]$. Этот метод рассмотрен в $[20,21]$, метод тета-функции предложен в [25] и развит в [26].
Методы вычисления интеграла $I_{2}$ пока не опубликованы (по сведениям автора). Для вычисления $I_{2}$ предлагается аппроксимировать функцию $\Phi_{\perp}(\lambda)$, как и при вычислении $I_{1}$, интерполяционным многочленом $\sum_{\nu=0}^{N} \exp \left(-v \lambda \tau_{0}\right) \approx \Phi_{\perp}(\lambda)$ на чебышевской сетке [27] и вычисление интеграла свести к вычислению суммы:

$$
\begin{aligned}
& \int_{0}^{\infty} J_{1}(\lambda r) \sum_{\nu=0}^{N} \exp \left(-\nu \lambda \tau_{0}\right) d \lambda \\
& =\frac{1}{r} \sum_{\nu=0}^{N}\left[1-\frac{v \tau_{0}}{\sqrt{r^{2}+\left(\nu \tau_{0}\right)^{2}}}\right] \approx I_{2}, \quad \tau_{0} \geq 0 .
\end{aligned}
$$

Аналитические выражения для математических моделей среды $\Phi, \Lambda, \Omega$, получаемые из граничных условий, достаточно громоздки; для повышения надежности результата (особенно при $m>4$ и/или $z_{0} \neq h$ ) систему уравнений, формируемую для получения $\Phi, \Lambda, \Omega$, целесообразно решать численно, при фиксированных значениях $\lambda$, соответствующих узлам интерполяции.

Приняв в качестве физической модели среды полупространства с плоской границей раздела и эквивалентными параметрами, по методу ЭПР с учетом формулы (11) функция Грина для поляризационного потенциала поля в плате при $\sigma=0$ :

$$
\Pi_{z_{1}}=\frac{\left(k_{e_{2}}^{2}-k_{e_{1}}^{2}\right) x}{\left(k_{e_{1}}^{2}+k_{e_{2}}^{2}\right) r^{2}}\left(1-\frac{z-z_{0}}{R_{1}}\right), \quad z-z_{0} \leq 0,
$$

где

$$
k_{e j}(r, z)=\omega \sqrt{\varepsilon_{0} \varepsilon_{e j} \mu_{0} \mu_{e j}}, \quad j=1,2 .
$$

Z-компонента поляризационного потенциала поля, создаваемого проводником-источником, рассчитывается интегрированием соответствующей функции Грина с весом, равным плотности тока, по объему этого проводника. Закон распределения плотности тока $j\left(x_{0}, y_{0}, z_{0}\right)$ по объему проводника и различные аппроксимации этого закона рассмотрены в [12,27]

$$
j\left(x_{0}, y_{0}, z_{0}\right)=I\left(x_{0}\right) j\left(y_{0}\right) j\left(z_{0}\right) \text {. }
$$

Решение уравнения Гельмгольца для тока в согласованной однородной линии произвольного сечения, ориентированной вдоль оси абсцисс, описывается хорошо известным выражением

$$
I\left(x_{0}\right)=I_{1} \exp \left(-\gamma\left|x_{0}-x_{s}\right|\right)
$$

где $I_{1}=U_{0} / \sqrt{\left(i \omega L+r_{0}\right) /\left(i \omega C+g_{0}\right)}-$ ток в начале линии (проводника-источника), т.е. при $x=x_{s}$; $\gamma=\sqrt{\left(i \omega L+r_{0}\right)\left(i \omega C+g_{0}\right)} / l_{s}-$ постоянная распространения электромагнитной волны вдоль однородной линии; $L, C, r_{0}, g_{0}$ - полные распределенные параметры линии (индуктивность, емкость, активное сопротивление и активная проводимость утечки соответственно). 
Распределение тока вдоль оси ординат $j\left(y_{0}\right)$ можно аппроксимировать $\delta$-функцией Дирака [12,П3]:

$$
j\left(y_{0}\right)=2 \delta\left(\left|y_{0}\right|-b / 2\right) .
$$

Распределение $j\left(z_{0}\right)$ можно считать равномерным. Тогда поляризационный потенциал помехонесущего поля, создаваемого проводником-источником в точке с координатами $\{x, y, z\}$, описывается формулой

$$
\Pi_{s}(x, y, z)=\int_{0}^{t} j\left(z_{0}\right) d z_{0} \int_{-b / 2}^{b / 2} j\left(y_{0}\right) d y_{0} \int_{x_{s}}^{x_{s}+l_{s}} I\left(x_{0}\right) \Pi_{z_{1}} d x_{0},
$$

где $x_{s}, x_{s}+l_{s}-$ абсциссы начала и конца проводникаисточника.

Практически без потери точности плотность тока можно принять постоянной по толщине и ширине проводника-источника. Тогда получим

$$
\Pi_{s}\left(x_{s}+l_{s}\right)=\frac{1}{t b} \int_{0}^{t} d z_{0} \int_{0}^{b} d y_{0} \int_{x_{s}}^{x_{s}+l_{s}} I\left(x_{0}\right) \Pi_{z_{1}} d x_{0} .
$$

Выразив потенциалы $\varphi$ и $A$ через вектор Герца, наводимую в проводнике-рецепторе ЭДС $\chi$ можно вычислить по формуле (1) через интеграл от вертикальной компоненты напряженности электрического поля $E_{z_{1}}$ при фиксированных значениях $x$ и $y$, соответствующих координатам рецептора:

$$
\chi=\int_{z_{1}}^{z_{2}} E_{z_{1}} d z=-\left[\left.\frac{\partial \Pi_{s}}{\partial z}\right|_{z=z_{2}}-\left.\frac{\partial \Pi_{s}}{\partial z}\right|_{z=z_{1}}\right]+k_{e_{2}}^{2} \int_{z_{1}}^{z_{2}} \Pi_{s} d z .
$$

Интеграл вычисляется численно. Расчет эквивалентных проницаемостей рассмотрен выше.

Задача решена.

\section{Список литературы}

[1] Франк Ф.Р., Мизес М. Дифференциальные и интегральные уравнения математической физики. Ч. 2. Л.: ОНТИ, 1937. $1000 \mathrm{c}$.

[2] Гринберг Г.А. Избранные вопросы математической теории электрических и магнитных явлений. М., Л.: АН СССР, 1948. $728 \mathrm{c}$

[3] Ming-Ju Tsai, Chinglung Chen, Alexopoulos N.G. // Electromagnetics. 1998. Vol. 18. N 3. P. 267-288.

[4] Jackson D.R., Alexopoulos N.G. // IEEE Transactions on Antenna Propagation. 1980. Vol. AP-34. N 12. P. 1467-1470.

[5] Балюк Н.В., Зеленин А.Н. // Технологии электромагнитной совместимости. 2006. № 2 (17). С. 54-58.

[6] Konefal T., Dawson J.F., Marvin A. // IEEE Transactions on Electromagnetic Compatibility. 2005. Vol. 47. N 4. P. 678-691.

[7] Wallyn W., De Zutter D., Rogier H. // IEEE Transactions on Electromagnetic Compatibility. 2002. Vol. 44. N 1. P. 130-138.

[8] Kochetov S.V. Time-and-frequency-domain modeling of passive interconnection structures in field and circuit analysis. Magdeburg: Magdeburger forum zur Elektrotechnik, 2008. $211 \mathrm{p}$.
[9] Antonini G., Deschrijver D., Dhaene T. // IEEE Transactions on Electromagnetic Compatibility. 2007. Vol. 49. N 1. P. 35-48.

[10] Ren K., Railton C.J. // IEEE Transactions on Antennas and Propogation. October 2008. Vol. 56. N 10. P. 3253-3259.

[11] Конников И.А. // ЖТФ. 2013. № 10. С. 8-12.

[12] Конников И.А. // Электромагнитные волны и электронные системы. 2013. № 7. С. 53-60.

[13] Конников И.А. // Информационные технологии. 2013. № 4. C. 2-8.

[14] Конников И.А. // Вестник МГТУ им. Н.Э. Баумана, сер. Приборостроение. 2007. № 4(69). С. 3-20.

[15] Sommerfeld A. Partial differential equations in physics. N. Y.: Academic Press Inc., 1949. 335 p.

[16] Конников И.А. // Математическое моделирование. 2007. T. 19. № 4. C. 37-44.

[17] Стрэттон Джс.А. Теория электромагнетизма. М., Л.: ОГИЗ, 1948. $540 \mathrm{c}$.

[18] Лавров Г.А., Князев А.С. Приземные и подземные антенны. М.: Сов. радио, 1965. 260 с.

[19] Конников И.А. // Петербургский журнал электроники. 2013. № 3. С. 97-104.

[20] Конников И.А. // Прикладная физика и математика. 2014. № 3. С. 39-50.

[21] Конников И.А. // Прикладная физика. 2007. № 2. С. 17-24.

[22] Куфуд O. Зондирование методом сопротивлений. М.: Недра, 1984. $270 \mathrm{c}$.

[23] Говорков В.А. М., Л.: Госэнергоиздат, 1960. 464 с.

[24] Тихонов А.Н., Самарский А.А. Уравнения математической физики. М.: Наука, 1977. 736 с.

[25] Конников И.А. // Электричество. 2007. № 3. С. 37-41.

[26] Конников И.А. // ЖТФ. 2007. Вып. 1. С. 15-20.

[27] Конников И.А. // Прикладная физика и математика. 2013. № 6. С. $75-83$. 